\title{
Respiratory syncytial virus and airway microbiota - A complex interplay and its reflection on morbidity
}

\author{
Giovanni Rossi ${ }^{1}$, Stefania Ballarini ${ }^{2}$, Michela Silvestri $^{3}$, Oliviero $\mathrm{Sacco}^{3}$, and Andrew Colin ${ }^{4}$ \\ ${ }^{1} \mathrm{G}$. Gaslini Institute \\ ${ }^{2}$ University of Perugia \\ ${ }^{3}$ Istituto Giannina Gaslini \\ ${ }^{4}$ Director, Division of Pediatric Pulmonology
}

November 26, 2020

\begin{abstract}
The immunopathology of respiratory syncytial virus (RSV) infection, the most common cause of lower respiratory tract infections (LRTI) in the pediatric population, with severe disease being the exception. The variability of the clinical presentation is incompletely explained by host, viral and environmental factors but, in infants and young children, disease severity is certainly linked to the physiological immune immaturity. There is evidence that the maturation of the host immune response is, at least in part, promoted by the composition of the nasopharyngeal microbiome that, modulating excessive inflammation, can counteract the predisposition to develop viral respiratory infections and lower the risk of disease severity. However, interaction between the nasopharyngeal microbiota and respiratory viruses can be bidirectional. Microbial dysbiosis can drive disease pathogenesis but may also represents a reflection of the disease-induced alterations of the local milieu. Moreover, viruses like RSV, can also increase the virulence of potential pathogens in nasopharynx, which is a main reservoir of bacteria, and therefore promote their spread to the lower airways causing superinfection. Negative changes in microbial community composition in early life may constitute a heightened risk towards severe RSV respiratory infection and bacterial superinfection, whilst specific groups of microorganisms can be associated with protection. A better understanding into the potential negative and positive role of the different nasopharyngeal bacterial species in disease prevention as well as into the possible benefits of microbiome therapeutic manipulation, may improve patient outcomes.
\end{abstract}

Respiratory syncytial virus and airway microbiota - A complex interplay and its reflection on morbidity Giovanni A. Rossi ${ }^{1}$, Stefania Ballarini ${ }^{2}$, Michela Silvestri ${ }^{1}$, Oliviero Sacco ${ }^{1}$ and Andrew A. Colin ${ }^{3}$

${ }^{1}$ Department of Pediatrics, Pulmonary and Allergy Disease Unit, G. Gaslini University Hospital, Genoa, Italy, ${ }^{2}$ Department of Experimental Medicine, Section of Immunometabolism, Immunogenetics and Translational Immunology, University of Perugia, Italy, and ${ }^{3}$ Division of Pediatric Pulmonology, Miller School of Medicine, University of Miami, Miami, FL, USA.

E-mail addresses: giovannirossi@gaslini.org

Correspondence to. Giovanni A. Rossi, Department of Pediatrics, Pulmonary and Allergy Disease Pediatric Unit and Cystic Fibrosis Center, Istituto Giannina Gaslini, Genoa, Italy;- giovannirossi@gaslini.com

Abstract: 233 words; text: 4.382 words; reference: 55 ; figures 3

\section{Abstract}


The immunopathology of respiratory syncytial virus (RSV) infection, the most common cause of lower respiratory tract infections (LRTI) in the pediatric population, with severe disease being the exception. The variability of the clinical presentation is incompletely explained by host, viral and environmental factors but, in infants and young children, disease severity is certainly linked to the physiological immune immaturity. There is evidence that the maturation of the host immune response is, at least in part, promoted by the composition of the nasopharyngeal microbiome that, modulating excessive inflammation, can counteract the predisposition to develop viral respiratory infections and lower the risk of disease severity. However, interaction between the nasopharyngeal microbiota and respiratory viruses can be bidirectional. Microbial dysbiosis can drive disease pathogenesis but may also represents a reflection of the disease-induced alterations of the local milieu. Moreover, viruses like RSV, can also increase the virulence of potential pathogens in nasopharynx, which is a main reservoir of bacteria, and therefore promote their spread to the lower airways causing superinfection. Negative changes in microbial community composition in early life may constitute a heightened risk towards severe RSV respiratory infection and bacterial superinfection, whilst specific groups of microorganisms can be associated with protection. A better understanding into the potential negative and positive role of the different nasopharyngeal bacterial species in disease prevention as well as into the possible benefits of microbiome therapeutic manipulation, may improve patient outcomes.

\section{INTRODUCTION}

Respiratory syncytial virus (RSV) is the main cause of acute viral respiratory infection leading to hospitalization in infants and young children worldwide [1]. The spectrum of its clinical manifestations ranges from mild upper tract illness to severe LRTI. The latter, requiring hospitalization in approximately $20 \%$ of the cases and of these $\sim 15 \%$ require intensive care management [2]. RSV virulence and a variety of environmental factors contribute to increased RSV susceptibility, as well, specific host related conditions have been identified which increase the risk for severe disease [1-3], yet these are not present in most infants hospitalized with RSV LRTI. The mechanisms accounting for the wide variability in the clinical presentation of RSV infection are only partly understood, but recent insights suggest that modifications of the nasopharyngeal microbiota composition established or induced by a previous infection might be involved. In early life, the baseline system structure and function of the airway immunity is regulated by the local microbial milieu, and distinct microbiota have been associated with sequelae of specific viral and bacterial LRTI [4-6]. Recent insights suggest that modifications of the nasopharyngeal microbiota composition, established by a preceding infection, might be involved. Whether imbalance in the microbial community can drive disease pathogenesis or, conversely, reflects disease-induced alterations of the local milieu following respiratory infections is still to be elucidated $[5,6]$. A paradigmatic example is represented by the bidirectional interplay between resident nasopharyngeal bacterial communities and RSV in bronchiolitis. Modifications of the upper airway microbiota composition appears to be associated with RSV disease severity, at the same time there is evidence that RSV can increase the virulence of potential nasopharyngeal pathogens, a large bacterial reservoir, that can consequently spreads to the lower airways causing superinfection $[7,8]$. The current evidences on the epidemiologic link between RSV and nasopharyngeal microbiome, the mechanisms involved in these interactions, and the possible clinical consequences will be reviewed, summarized and discussed.

\section{INFANT NASOPHARYNGEAL MICROBIOME IN HEALTH AND DISEASE}

The airways represent a large surface area that interacts with the surrounding environment. They exhibit a gradient of bacterial load that is relatively high in the nasopharynx and substantially diminished in the lower respiratory tract [1]. A growing body of literature has demonstrated that the nasopharyngeal microbiome, with its mixed microbial communities, plays an important role in maturation and homeostasis of the host immune response in the upper and lower airways $[1,4,5]$. The balanced competitive/synergistic interplay between commensal and potentially pathogenic taxa, that is the hallmark of a healthy status, can be altered by acquisition of new pathogenic bacteria, increased virulence or prevalence of potential pathogens, and/or decreased efficiency of host defenses, the latter being the cause but also the result of dysbiosis $[1,4,6]$. In newborns and infants, obligatory nasal breathers, nasopharyngeal airways are both the first line of defense but also the main port of entry for bacteria and viruses. Overgrowth of virulent taxa will 
lead to symptomatic infection, with surrounding tissue invasion and inflammation. [9]. Therefore, in early life, microbiota composition of nasopharyngeal airways reflects exposures to the surrounding atmospheric environment which, shaping the host immune responses, will favor or inhibit the predisposition to respiratory disorders $[1,4,10-12]$. Nasopharyngeal colonization by environmental potential respiratory pathogens is established early in childhood [2]. In a study on 234 infants, it was shown that nasopharyngeal microbiome has a relatively simple structure, dominated by six clusters: Moraxella ,Haemophilus (Gram negative bacteria), Staphylococcus ,Corynebacterium, Streptococcus and Alloiococcus(Gram positive bacteria) (figure 1) [13]. Healthy nasopharyngeal samples collected around 2 months of age were dominated byStaphylococcus and Corynebacterium, but the frequency of these microbiome profile groups declined with age and, at 12 months of age, an increased prevalence of Alloiococcus and Moraxellaspecies (spp) was detected (figure 1A) [13]. Transient incursions ofStreptococcus, Moraxella and/or Haemophilusoccurred during acute viral respiratory tract infections,Streptococcus and Moraxella colonizing, respectively, $54 \%$ and $72 \%$ of the children by 1 year of age [14] and a progressive decline in Staphylococcus and Corynebacterium spp (figure 1B) [13]. Antibiotic treatments were associated with higher abundances of the potential respiratory pathogens Haemophilus, Streptococcus andMoraxella, with lower abundances of Alloiococcus and Corynebacterium (figure 1C) [13]. This microbial dysbiosis may predict subsequent respiratory infections [2,15-17]. Colonization with Streptococcus, Moraxella orHaemophilus spp was linked to an increased risk for LRTI, whilst nasopharyngeal microbiota dominated by Corynebacterium or Alloiococcus clusters was associated with less respiratory morbidity (figure 1D) [11-13]. When related to early exposure to noxious environmental factors negative changes in microbial community composition may constitute a heightened risk towards severe viral respiratory infections and bacterial superinfections $[11,17]$. This can be observed in severely premature infants admitted to neonatal intensive care units (NICU). These infants face the combined effects of deprivation of the normal intrauterine physical environment, supplemental oxygen with relative hyperoxia, barotrauma from mechanical respiratory support, exposure to nosocomial pathogens, and broad-spectrum antimicrobials [16]. They show abnormal nasal airway immune responses against respiratory viruses, persisting beyond the PICU period and, in comparison with full term infants, a higher nasopharyngeal microbial heterogeneity and within-group dissimilarity [18]. Emergence of a mixed flora, including Moraxella and other Gram-negative bacteria (Burkholderia, Neisseria and Janthinobacterium ), was detected. At phylum level, an increase in Proteobacteria (a major group of Gram-negative bacteria) was associated with a decrease inFirmicute (Gram-positive bacteria such as Lactobacillus ) (figure 1E) [14]. This airway microbiome signature has been recently described also in preterm newborns at increased risk of bronchopulmonary dysplasia [16,19]. The presence of within-group dissimilarity, identified also in the nasopharyngeal microbiota of otherwise healthy premature infants, suggests that this group does not develop a stable microbiome in early life. Longitudinal data showed that these prematurity-related microbiota features persisted during viral infection and played important negative role in modulating airway inflammatory and immune responses in this vulnerable group $[16,19]$. Upper airways colonization by potential pathogens in neonates, also in term infants, may be associated with increased susceptibility to LRTI during the first 3 years of life. A prospective birth cohort study of 411 children born to mothers with asthma, showed that neonatal hypopharyngeal colonization with Streptococcus pneumoniae, Haemophilus influenzae, orMoraxella catarrhalis, at age 4 weeks, was associated with increased risk of bronchiolitis, recurrent wheeze, asthma and pneumonia, during the first 3 years of life [20,21]. The viral etiology of bronchiolitis, i.e. whether it was RSV or human rhinovirus (HRV) related, was not reported in this study.

\section{NASOPHARYNGEAL MICROBIOTA COMMUNITY COMPOSITION AND RSV IN- FECTION}

\subsection{Modulation of RSV infection severity}

In infants, RSV bronchiolitis severity is linked to a dysregulation of the immune response, related to a relative immaturity of the host defenses [3]. Recent literature suggests that prior or concomitant colonization of nasopharyngeal microbiota by potentially pathogenic bacteria may enhance the severity of the RSV infection $[7,22,23]$. A 17-site, multicenter, prospective cohort study of 1,016 infants (age $<1$ year) hospitalized with bronchiolitis, showed that during RSV infection a greater abundance of Haemophilus influenzae 
andStreptococcus pneumoniae in the nasal microbiota can be associated with more severe disease (figure $2 \mathrm{~A}$ ) [22]. In a prospective observational study, during four consecutive seasons, 106 young children with a first episode of RSV infection and 26 healthy control subjects were enrolled [7]. Three groups of children less than 2 years of age were evaluated: a) with mild RSV disease (RSV infection), b) with severe RSV disease (RSV hospitalization) and c) healthy controls. Five nasopharyngeal microbiota clusters were identified, characterized by enrichment of either Haemophilus influenzae, Streptococcus, Corynebacterium, Moraxella, and Staphylococcus aureus [7]. RSV infection and hospitalization were positively associated with Haemophilus influenzae and Streptococcus and negatively associated withStaphylococcus aureusabundance (figure 2A). Rates of PICU admission and length of stay tended to be lower in children within the Moraxellacluster than in children included in the other clusters and higher abundance of Moraxella was observed most often in outpatients [7]. Independently from microbiota cluster, children with RSV infection showed overexpression of IFN-related genes. However, in those with transcriptome profiles dominated by Haemophilus influenzaeand Streptococcus, an upregulation of genes linked to toll-like receptor and neutrophil-macrophage activation and signaling was also detected. Overexpression of Haemophilus and loss in commensals, like Veillonella, were described in nasopharyngeal aspirates of a cohort of 54 infants, younger than 6 months of age, hospitalized with RSV infection [24]. Transcriptome profiles dominated by Haemophilus was associated with increase IL-6 and CXCL-8 responses, cytokines that in respiratory virus infections are associated with more severe disease (figure 2A). [24-26]. Moreover, in human bronchial epithelial cell culture pretreated with Haemophilus influenzae, the release of IL-6 and CXCL-8 was synergistically increased after RSV, but not after HRV infection, buttressing the notion that this virus-bacterial interplay is specific for RSV [27]. The clinical implication of these observation is reflected in a multicenter prospective cohort study of 1,005 infants hospitalized for bronchiolitis. In this study the odds ratio for PICU admission was higher for subjects with RSV infection and Haemophilus -dominant profiles compared to RSV-infected infants with profiles dominated by other bacteria, such as Moraxella(figure 2A) [28]. Thus, specific nasopharyngeal microbiota clusters, dominated by Haemophilus influenzae and Streptococcus pneumoniae can modulate the host immune response, potentially affecting clinical disease severity. Further evidence for the ability of the nasopharyngeal microbiota to positively modulate the host immune response is the demonstration of the presence of specific metabolic profiles, linked to the microbiome composition, in children with severe bronchiolitis. In a multicenter prospective cohort study on infants $<1$ year of age, hospitalized with bronchiolitis, Stewart et al. determined microbiome profiles and metabolome in nasopharyngeal airway samples and their association with disease severity [22]. Among 254 metabolites identified, a panel of 25 showed high sensitivity (84\%) and specificity (86\%) in predicting the use of positive pressure ventilation (PPV) support. Metagenomic sequencing demonstrated that Haemophilus influenzae, Moraxella catarrhalis, and Streptococcus pneumoniae dominated the nasopharyngeal airway. Of these, however, only the relative abundance of Streptococcus pneumoniaecorrelated with the intensity of these selected metabolites to predict increased risk of PPV support (figure 2A). In the pathway analysis, sphingolipid metabolism was the sub-pathway most significantly enriched in infants with PPV use, and in turn, enrichment of sphingolipid metabolites was positively correlated with the relative abundance ofStreptococcus pneumoniae [22]. In contrast, the relative Moraxella abundance was negatively correlated with respective intensity of the same metabolites. Sphingolipids are linked to the inflammatory-mediated pathogenesis of airway diseases and play an important role in multiple aspects of viral replication, including the initial infection of mammalian cells, depression of host immune response, and assembly and budding of newly synthesized viral components [29]. Thus, curiously, Moraxella spp, thought to be associated with a general increase in infant morbidity [11,13], appears to have a "protective effect" on RSV bronchiolitis.

\subsection{Delayed viral clearance, RSV and HRV infection and recurrent wheezing}

Respiratory virus clearance requires a complex response initiated by resident respiratory tract cells and innate immune cells and ultimately resolved by adaptive immune cells [30]. This viral clearance that is largely dependent on the interplay between infecting agents and host immune response, may be influenced by nasopharyngeal microbiota composition in infants with RSV bronchiolitis. A 17-center prospective cohort study of infants aged less than 1 year, with RSV bronchiolitis, found that those with a nasopharyngeal Haemophilus -dominant microbiota profile at time of hospitalization were more likely than those with a 
mixed profile to have delayed viral clearance, also after adjustment for 11 factors, including viral load (figure 2B) [23]. Nasopharyngeal microbiota composition at the time of hospitalization was also described as associated to an increased risk of recurrent wheezing. In children $<2$ year of age, with severe bronchiolitis due to RSV and/or HRV enrichment of Haemophilus influenzae and Moraxella catarrhalis was found in their nasopharynx (figure 2B) [31]. Haemophilus influenzae was detected in most RSV-only infected and co-infected samples, but not in HRV-only samples, whilst Moraxella spp were detected in co-infection and were more common among children with wheezing upon admission [31]. No enrichment of Haemophilus influenzae in HRV-only and of Moraxella spp in RSV- or HRV-only was reported. It is therefore possible that specific bacterial species increase the likelihood of one viral infection or another, and play a role in the onset of wheezing (figure 2B). This may be the case of the association of Moraxella spp enrichment with HRV infection. In 17 U.S.A. centers, nasal swabs were collected in infants hospitalized for bronchiolitis and repeated 3 weeks and 1 year after hospitalization [32]. The viral etiology of bronchiolitis was not reported. An increase in relative abundance of Moraxella orStreptococcus spp, at 3 weeks, and of Streptococcus spp, at 1-year follow-up was associated with increased risk of recurrent wheezing in the study subjects [32]. In a similar study performed in Beijing Children's Hospital; 74 infants aged 6 months or less, hospitalized for an initial episode of severe RSV bronchiolitis, were included and followed until age 3 years [33]. A higher relative abundance of Haemophilus, Moraxella and Klebsiellawas detected in nasotracheal aspiration in the 26 infants $(35.1 \%)$ who later developed recurrent wheezing. In these infants, higher abundance of Haemophilus or of Moraxella was respectively associated with elevated CXCL8 levels or of IL-6 and IL-10 (figure 2B). Resolving the conundrum of the differential role of Moraxella, as having an apparent protective role on the severity of RSV infection in the acute phase, yet being consistently identified as a risk pathogen for recurrent wheezing, will advance our comprehension on both its role and that of other nasopharyngeal microbial species' in the course, severity and complications of different respiratory conditions. A different nasopharyngeal microbiota composition was observed as associated with a reduced risk of wheeze after RSV infection. A population-based birth cohort of 118 previously healthy term infants was evaluated during the first confirmed RSV acute respiratory infection in a Tennessee-based study [34]. Of the $113(95.8 \%)$ children who had 2-year outcome data, $46(40.7 \%)$ had at least one parental reported wheezing episode. There was no association between the nasopharyngeal microbiome taxonomic composition, diversity and richness assessed both during the first RSV infection and the subsequent wheeze development. However, detection as well as relative abundance ofLactobacillus in nasopharyngeal aspirate was consistently higher in infants who did not develop wheezing. Lactobacillus also ranked first among the different genera in a model distinguishing infant with and without subsequent wheeze [34]. Increasing evidence supports that dysbiosis, in the first years of life may also have a role in the development of allergy and asthma because of the "gutlung axis" interaction. Therefore, probiotics were suggested as a possible therapeutic approach in prevention of wheezing episodes [35]. However, in a randomized double-blind study conducted in 131 young children (6-24 months old) with at least two wheezing episodes and a first-degree family history of atopic disease, 6 months treatment with oral Lactobacillus rhamnosus showed no clinical efficacy on asthma-related events and only mild effects on allergic sensitization which persisted 6 months after its cessation [36]. In a similar study of 160 children and adolescents (6-18 years old), with asthma diagnosis, 3 months of treatment with other species of probiotics,Lactobacillus paracasei, Lactobacillus fermentum, as well as their combination decreased IgE levels, and improved asthma control based on the Childhood Asthma Control Test, Pediatric Asthma Quality of Life Questionnaire scores, and improved peak expiratory flow rates[37]. Further studies are needed to confirm these inconsistent findings and, importantly, to investigate the possible long-term benefits of some probiotics in infants and young children at high risk for allergic sensitization, recurrent wheezing and asthma.

\section{NASOPHARYNGEAL MICROBIOTA COMMUNITY COMPOSITION AND BACTE- RIAL SUPER-INFECTION DURING RSV INFECTIONS}

The interaction between nasopharyngeal microbiota and RSV in children can be bidirectional. As discussed above, specific nasopharyngeal microbiota compositions may modulate RSV severity but, at the same time, 
RSV-induced infections can stimulate proliferation of potentially pathogenic microorganisms in the upper airways and thus favor bacterial superinfections and morbidity $[7,38,39]$. RSV infection can promote the invasion of the respiratory epithelium by non-typeable Haemophilus influenzae andStreptococcus pneumoniae through a variety of mechanisms, including upregulation of bacterial adhesion protein expression, loss of epithelial barrier integrity and decrease of bacterial clearance mechanisms [3,8,40,41]. Using an in vitro and a lung mouse model of infection, incubation with RSV or purified G protein was shown to increase Streptococcus pneumoniae adherence to differentiated ciliated cells of the respiratory epithelium, thus enhancing the severity of the airway infection and decreasing animal survival [8,42]. Also detected in these studies were extensive changes in the pneumococcal transcriptome, with upregulation in the expression of key pneumococcal virulence genes, including those encoding the pneumococcal toxin pneumolysin [42]. On the airway epithelium end, RSV infection upregulated the cellular surface expression of other known receptors for Streptococcus pneumoniaeand non-typable Haemophilus influenzae, notably the intercellular adhesion molecule-1 (ICAM-1) and platelet-activating factor receptor [8]. In clinical practice, for infants with RSV bronchiolitis the risk of secondary bacterial infections (SBI) is present, yet the severity of the resulting bacterial infection appears to parallel that of the RSV infection (figure 3A). The low risk of SBI in mild RSV is born out in a study on 1,248 febrile infants $<60$ days of age, from 88 pediatric Emergency Departments in New York. The rate of SBI was significantly lower in those with RSV infection (7.0\%) than in those without RSV infection (12.5\%) [43]. Additionally, as compared to the RSV-negative infants, RSV-positive infants had a lower rate of bacteremia (1.1\% vs 2.3$)$ and of urinary tract infections (UTI) (5.4\% vs $10.1 \%$ ). Similar results were reported in a retrospective cohort study of $<8$-week-old infants, who presented with fever and a positive RSV antigen test to the Emergency Department at an urban child facility in Nashville, Tennessee [44]. Buttressing these results is a systematic review of 11 studies on "in- and out-patients", < 60 to 90 day old, with bronchiolitis, reporting no case of bacteremia, yet the rate of UTI resulting slightly higher in the RSV-positive subgroup (5.1\% vs $2.0 \%$ ) [45]. These data suggest that, in nontoxic-appearing infants with a positive RSV test, full septic evaluations are not necessary, however, the finding of a low, but clinically relevant rate of urinary tract infection, probably justifies urine work up $[44,45]$. Conversely, the incidence of SBI and concomitant bacterial pneumonia is more frequently observed (or diagnosed) in infants with severe RSV bronchiolitis (figure 3B). Out of 127 infants (median age 1.7 months) admitted to the PICU of the Zurich University Children's Hospital, 57 were mechanically ventilated [46]. Tracheal cultures obtained from intubated children within the first hour of intubation were positive in 25 infants, half of them for community and half for nosocomial acquired bacteria. Microorganisms associated with bacterial LRTI included Moraxella catarrhalis ,Haemophilus influenzae type b and Streptococcus pneumoniae. The incidence of sepsis was $3 / 127$ patient. Despite only documenting 25 bacterial concomitant infection, antibiotics were administered to 73/127 infants over the first two days of hospital admission, namely, to a high proportion of those with negative bacterial cultures. The authors conclude that to avoid inappropriate antibiotic prescription, tracheal aspirates should be investigated microbiologically on a routine basis in this patient group [46]. The high frequency of bacterial coinfection in young infants with severe RSV bronchiolitis was confirmed in the study of the Royal Liverpool Children's Hospital [47]. Out of the 165 children (median age 1.6 months) admitted to PICU with RSV bronchiolitis, 70 (42.4\%) lower airway secretions tested positive for bacteria, including Haemophilus influenzae, Moraxella catarrhalis ,Streptococcus pneumoniae and Staphylococcus aureus[47]. All those with bacterial co-infection were mechanically ventilated and required ventilatory support for longer than those with only RSV. Gender, co-morbidity, origin, prior antibiotics, time on preceding antibiotics, admission oxygen, and ventilation index were not predictive of positive bacterial cultures [47]. The risk of prolonged hospitalization and PICU admission may be even higher in preterm born infants, hospitalized with RSV disease, if concurrent bacterial infections are present. Out of a total of 464 infants, median age 2.75 months (range 0.25-96), hospitalized due RSV infection to the Graz Medical University, 42 $(9.1 \%)$ were born $<37$ weeks of gestational age [48]. Concurrent bacterial infections were detected in $3.1 \%$ of term and $9.5 \%$ of preterm infants and the percentage of those referred to PICU was $5.2 \%$ and $23.8 \%$ in term and preterm infants respectively. In all these preterm a diagnosis of pneumonia was made, and tracheal aspirates were positive for Streptococcus pneumoniaeand Haemophilus influenzae. Mean hospital stay was longer (22.3 days) in preterm infants with bacterial co-infection than in the ones without it (10.3 days) [48]. 
Concomitant bacterial infection can occur also in older children hospitalized with RSV bronchopulmonary infections as shown in a study performed of 188 pediatric inpatients, aged up to 5 years, admitted to 2 hospitals in Chiba Prefecture, Japan [49]. Pathogenic bacteria were isolated from washed sputum bacterial culture in $43.6 \%$ of the children, the three most frequently isolated being Haemophilus influenzae (43.9\%), Streptococcus pneumoniae (36.6\%) and Moraxella catarrhalis (29.3\%). Before admission, $28.7 \%$ of the patients received oral antibiotics, most often macrolides and amoxicillin. Of the Haemophilus influenzae strain isolated $38.9 \%$ were ampicillin-resistant, whilst allStreptococcus pneumoniae strains were penicillin G sensitive, minimum inhibitory concentration values were $2 \mu \mathrm{g} / \mathrm{ml}$ [49]. Thus, RSV bronchopulmonary infection in hospitalized children can often be associated with super-infection by antimicrobial-resistant bacterial. Prior antibiotic treatment and Gram-negative pathogen prevalence at nasopharyngeal level can be associated with more disease severity. A prospective, observational cohort study, of 136 infants with RSV bronchiolitis enrolled at $24 \mathrm{~h}$ of hospitalization; 104 on the ward and 32 in the PICU [50]. As compared to an age-matched group of healthy controls, RSV bronchiolitis infants had more frequent nasopharyngeal colonization withnontypable Haemophilus influenzae or Moraxella catarrhalis (Gram-negative bacteria) andStaphylococcus aureus andStreptococcus pneumoniae (Gram-positive bacteria). Higher proportion of Gram-negative pathogens was associated with higher blood neutrophil percentages, greater number of nasal wash leukocytes, increased concentrations of plasma IL-8 and IL-6, a trend towards increased disease severity and longer need for oxygen. Thus,Staphylococcus aureus, which seems to have a protective role? on RSV infection, may be detected in the lower airways of young infants with severe RSV bronchiolitis but its presence does not seem to be associated with increase disease severity. In this study, $47 \%(64 / 136)$ of all RSV infants and $75 \%$ of those in PICU received antibiotics prior to sample collection. This common use of antibiotic was associated with a decreased likelihood of recovering potentially pathogenic bacteria by culture, but also with enhanced disease severity as defined by clinical parameters and radiologic findings. Infants treated with antibiotics prior to hospitalization required oxygen for a longer time and radiographic lobar consolidation was more frequently identified compared to those with no prior antibiotics. Atelectasis or lobar consolidation was associated with higher clinical disease severity scores [50]. The elevated antimicrobial-resistance in bacterial superinfection and the higher risks of enhanced disease severity highlight the need for microbiological investigation of airway secretion in this patient population. Finally, the incidence of Streptococcus pneumoniaecoinfection could be modified following the introduction of seven-valent pneumococcal conjugate vaccine (PCV7). Weinberger DM et al, evaluating hospitalization data from community hospitals in 18 U.S.A. states and comparing the 1992/1993 and 2008/2009 seasons, demonstrated a significant decline in RSV-coded hospitalizations in children aged $<1$ y and 1-2-year-old following PCV7 introduction in 2000 [51]. We could find no data on the effect of the effect of the subsequent introduction of PCV13.

\section{NASOPHARYNGEAL BACTERIAL COMMUNITY AS A SHIELD AGAINST RESPI- RATORY INFECTIONS}

Nasopharyngeal bacteria may counteract bacterial or viral infection. In infant mice, nasal priming with viable Corynebacterium pseudodiphtheriticum, a Gram-positive bacterium member of the normal microbiota of the upper respiratory tract, improved their resistance to primary RSV infection and secondary pneumococcal pneumonia [52]. The protection against RSV infection and pneumococcal superinfection was related to modulation of Toll-like receptor 3 activation in the respiratory tract leading to enhanced production of the protective cytokines TNF- $\alpha$, IL- 6 , IFN- $\gamma$ and IFN- $\beta$, but also of IL-10 that could contribute to restrict the inflammatory reaction and ultimately to limit tissue injury. IFN- $\gamma$ producing CD3+CD4+ cells were also detected in the lungs of treated infant mice, viral load was significantly lower and lung tissue damage markers (LDH and albumin concentrations) in bronchoalveolar lavage were decrease in RSV-infected mice. Priming with viable Corynebacterium pseudodiphtheriticumalso reduced lung bacterial cell counts and prevented the dissemination of S. pneumoniae into the blood [52]. Thus, a possible perspective use of these results could be a seasonal application of a nasal probiotic spray to boost respiratory innate immunity in immunocompetent subjects. Bearing in mind the "gut-lung axis" interaction, one could consider orally given probiotics or bacterial derived products as alternative options; a fascinating proposal whose theoretical efficacy needs eventually to be demonstrated by further studies [53]. A possible approach can be to assess bacterial lysate 
in germ free mice models to determine the effect on immune maturation and the capacity to mimic the microbiota effect in respiratory virus infection.

\section{CONCLUSIONS}

The nasopharyngeal microbiome plays an important role in modulating the susceptibility to respiratory infections in infants and young children. However, due to the complexity of the pathogenetic mechanisms involved, the role of dysbiosis at the nasopharyngeal levels on the pathogenesis of viral infections is still not completely understood. Recent insights have shown that early negative changes in microbial community composition, due to exposure to noxious environmental factors, may constitute a heightened risk for severe RSV respiratory infection as well as bacterial superinfection, in both term and preterm infants $[2,10,15,16]$. An exaggerated and ineffective inflammatory response to infection, associated with delayed viral clearance and increased risk of recurrent wheezing in later life are also observed [22]. The risk of serious bacterial superinfection, low in infants with mild RSV bronchiolitis, becomes higher when hospital admission with intensive care is required [46-50]. The elevated antimicrobial-resistance observed during bacterial super infections mandates careful stewardship and avoidance of inappropriate antibiotic prescription. Antimicrobial treatment does not eradicate bacteria from the nasopharynx. The reduction in carriage is temporary, followed by a rapid replacement, with overgrowth of more resistant strains, newly acquired or previously masked by other organisms $[2,8,54]$. The finding that specific groups of microorganisms might be associated with protection against RSV infection and less disease severity [28] suggests that studies should be done to understand the mechanistic insight into the potential positive role of these species in disease prevention. Further studies are also needed to investigate the possible short and long-term benefits of microbiome therapeutic manipulation and immune training with the goal of improving patient outcomes.

\section{References.}

1. Murphy T, Bakaletz L, Smeesters P. Microbial interactions in the respiratory tract. Pediatr Infect Dis J . 2009;28:S121-S126.

2. Garcia-Rodriguez JA, Fresnadillo Martinez MJ. Dynamics of nasopharyngeal colonization by potential respiratory pathogens.J Antimicrob Chemother . 2002;50 Suppl S2:59-73.

3. Rossi GA, Colin AA. Respiratory syncytial virus-host interaction in the pathogenesis of bronchiolitis and its impact on respiratory morbidity in later life. Pediatr Allergy Immunol . 2017;28:320-331.

4. Dickson RP, Erb-Downward JR, Martinez FJ, et al. The microbiome and the respiratory tract. Annu Rev Physiol 2016; 78: 481-504.

5. Gosens R, Hiemstra PS, Adcock IM, et al. Host-microbe cross-talk in the lung microenvironment: implications for understanding and treating chronic lung disease. Eur Respir J . 2020; 56:1902320.

6. Sakwinska O, Bastic Schmid V, Berger B, et al. Nasopharyngeal microbiota in healthy children and pneumonia patients. J Clin Microbiol . 2014;52:1590-1594.

7. de Steenhuijsen Piters WA, Heinonen S, Hasrat R, et al. Nasopharyngeal microbiota, host transcriptome, and disease severity in children with respiratory syncytial virus infection. Am J Respir Crit Care Med . 2016;194:1104-1115.

8. Rossi GA, Fanous H, Colin AA. Viral strategies predisposing to respiratory bacterial superinfections. Pediatr Pulmonol . 2020;55:1061-1073.

9. Bergeson PS, Shaw JC. Are infants really obligatory nasal breathers? Clin Pediatr (Phila) . 2001;40:567569.

10. Lal CV, Travers C, Aghai ZH, et al. The airway microbiome at birth.Sci Rep . 2016;6:31023.

11. Biesbroek G, Tsivtsivadze E, Sanders EA, et al. Early respiratory microbiota composition determines bacterial succession patterns and respiratory health in children. Am J Respir Crit Care Med . 2014; 190:1283-92.

12. Mika M, Mack I, Korten I, et al. Dynamics of the nasal microbiota in infancy: a prospective cohort study. J Allergy Clin Immunol . 2015; 135:905-12.e11.

13. Teo SM, Mok D, Pham K, et al. The infant nasopharyngeal microbiome impacts severity of lower respiratory infection and risk of asthma development. Cell Host Microbe . 2015;17:704-715. 
14. Faden H, Duffy L, Wasielewski R, Wolf J, Krystofik D, Tung Y. Relationship between nasopharyngeal colonization and the development of otitis media in children. Tonawanda/Williamsville Pediatrics. $J$ Infect Dis . 1997;175:1440-1445.

15. Dubourg G, Edouard S, Raoult D. Relationship between nasopharyngeal microbiota and patient's susceptibility to viral infection.Expert Rev Anti Infect Ther . 2019;17:437-447

16. Perez GF, Pérez-Losada M, Isaza N, Rose MC, Colberg-Poley AM, Nino G. Nasopharyngeal microbiome in premature infants and stability during rhinovirus infection. J Investig Med . 2017;65:984-990.

17. Følsgaard NV, Schjørring S, Chawes BL, et al. Pathogenic bacteria colonizing the airways in asymptomatic neonates stimulates topical inflammatory mediator release. Am J Respir Crit Care Med . 2013; 187:589-95.

18. Perez GF, Pancham K, Huseni S, et al. Rhinovirus-induced airway cytokines and respiratory morbidity in severely premature children.Pediatr Allergy Immunol . 2015; 26:145-52.

19. Segura-Cervantes E, Mancilla-Ramírez J, González-Canudas J, et al. Inflammatory response in preterm and very preterm newborns with sepsis. Mediators Inflamm . 2016; 2016:6740827.

20. Bisgaard H, Hermansen MN, Buchvald F, Loland L, Halkjaer LB, Bønnelykke K, Brasholt M, Heltberg A, Vissing NH, Thorsen SV, Stage M, Pipper CB. Childhood asthma after bacterial colonization of the airway in neonates. $N$ Engl $J$ Med . 2007;357:1487-95.

21. Vissing NH, Chawes BL, Bisgaard H. Increased risk of pneumonia and bronchiolitis after bacterial colonization of the airways as neonates.Am J Respir Crit Care Med . 2013; 188:1246-52.

22. Stewart CJ, Mansbach JM, Wong MC, et al. Associations of nasopharyngeal metabolome and microbiome with severity among infants with bronchiolitis. A multiomic analysis. Am J Respir Crit Care Med . 2017;196:882-891.

23. Mansbach JM, Hasegawa K, Piedra PA, et al. Haemophilus-dominant nasopharyngeal microbiota is associated with delayed clearance of respiratory syncytial virus in infants hospitalized for bronchiolitis. J Infect Dis . 2019;219:1804-1808.

24. Ederveen THA, Ferwerda G, Ahout IM, et al. Haemophilus is overrepresented in the nasopharynx of infants hospitalized with RSV infection and associated with increased viral load and enhanced mucosal CXCL8 responses. Microbiome . 2018;6:10.

25. Pyle CJ, Uwadiae FI, Swieboda DP, Harker JA. Early IL-6 signalling promotes IL-27 dependent maturation of regulatory $\mathrm{T}$ cells in the lungs and resolution of viral immunopathology. PLoS Pathog. 2017;13:e1006640.

26. Openshaw PJM, et al. Protective and harmful immunity to RSV infection. Annu Rev Immunol. 2017;35:501-32.

27. Gulraiz F, Bellinghausen C, Bruggeman CA, Stassen FR. Haemophilus influenzae increases the susceptibility and inflammatory response of airway epithelial cells to viral infections. FASEB J . 2015;29:849858.

28. Hasegawa K, Mansbach JM, Ajami NJ, et al. Association of nasopharyngeal microbiota profiles with bronchiolitis severity in infants hospitalised for bronchiolitis.Eur Respir J . 2016;48:1329-1339.

29. Heung LJ, Luberto C, Del Poeta M. Role of sphingolipids in microbial pathogenesis. Infect Immun . 2006;74:28-39.

30. Hall CB, Douglas RG Jr, Geiman JM. Respiratory syncytial virus infections in infants: quantitation and duration of shedding. J Pediatr . 1976; 89:11-5.

31. Newton AH, Cardani A, Braciale TJ. The host immune response in respiratory virus infection: balancing virus clearance and immunopathology. Semin Immunopathol . 2016;38:471-482.

32. Hyde ER, Petrosino JF, Piedra PA, Camargo CA Jr, Espinola JA, Mansbach JM. Nasopharyngeal Proteobacteria are associated with viral etiology and acute wheezing in children with severe bronchiolitis. J Allergy Clin Immunol 2014;133:1220-2.

33. Mansbach JM, Luna PN, Shaw CA, et al. Increased Moraxella and Streptococcus species abundance after severe bronchiolitis is associated with recurrent wheezing. J Allergy Clin Immunol . 2020;145:518527.e8.

34. Zhang X, Zhang X, Zhang N, et al. Airway microbiome, host immune response and recurrent wheezing 
in infants with severe respiratory syncytial virus bronchiolitis. Pediatr Allergy Immunol . 2020;31:281289.

35. Rosas-Salazar C, Shilts MH, Tovchigrechko A, et al. Nasopharyngeal Lactobacillus is associated with a reduced risk of childhood wheezing illnesses following acute respiratory syncytial virus infection in infancy. J Allergy Clin Immunol . 2018;142:1447-1456.e9.

36. Kozik AJ, Huang YJ. The microbiome in asthma: Role in pathogenesis, phenotype, and response to treatment. Ann Allergy Asthma Immunol . 2019;122:270-275.

37. Rose MA, Stieglitz F, Koksal A, Schubert R, Schulze J, Zielen S. Efficacy of probiotic Lactobacillus GG on allergic sensitization and asthma in infants at risk. Clin Exp Allergy . 2010;40:1398-1405.

38. Huang CF, Chie WC, Wang IJ. Efficacy of Lactobacillusadministration in school-age children with asthma: a randomized, placebo-controlled trial. Nutrients . 2018;10:1678.

39. Brealey JC, Sly PD, Young PR, Chappell KJ. Viral bacterial co-infection of the respiratory tract during early childhood.FEMS Microbiol Lett . 2015;362:fnv062.

40. Diaz-Diaz A, Garcia-Maurino C, Jordan-Villegas A, Naples J, Ramilo O, Mejias A. Viral bacterial interactions in children: impact on clinical outcomes. Pediatr Infect Dis J . 2019;38:S14-S19.

41. Avadhanula V, Wang Y, Portner A, Adderson E. Nontypeable Haemophilus influenzae and Streptococcus pneumoniae bind respiratory syncytial virus glycoprotein. J Med Microbiol. 2007;56:1133-7.

42. Jiang Z, Nagata N, Molina E, Bakaletz LO, Hawkins H, Patel JA. Fimbria-mediated enhanced attachment of nontypeable Haemophilus influenzae to respiratory syncytial virus-infected respiratory epithelial cells. Infect Immun . 1999;67:187-92.

43. Smith CM, Sandrini S, Datta S, et al. Respiratory syncytial virus increases the virulence of Streptococcus pneumoniae by binding to penicillin binding protein 1a. A new paradigm in respiratory infection. Am J Respir Crit Care Med . 2014;190:196-207.

44. Levine DA, Platt SL, Dayan PS, et al. Risk of serious bacterial infection in young febrile infants with respiratory syncytial virus infections. Pediatrics . 2004;113:1728-1734.

45. Titus MO, Wright SW. Prevalence of serious bacterial infections in febrile infants with respiratory syncytial virus infection. Pediatrics . 2003;112:282-284.

46. Ralston S, Hill V, Waters A. Occult serious bacterial infection in infants younger than 60 to 90 days with bronchiolitis: a systematic review. Arch Pediatr Adolesc Med . 2011;165:951-956.

47. Duttweiler L, Nadal D, Frey B. Pulmonary and systemic bacterial co-infections in severe RSV bronchiolitis. Arch Dis Child . 2004;89:1155-1157.

48. Thorburn K, Harigopal S, Reddy V, Taylor N, van Saene HK. High incidence of pulmonary bacterial coinfection in children with severe respiratory syncytial virus (RSV) bronchiolitis. Thorax . 2006;61:611615.

49. Resch B, Gusenleitner W, Mueller WD. Risk of concurrent bacterial infection in preterm infants hospitalized due to respiratory syncytial virus infection. Acta Paediatr . 2007;96:495-498.

50. Hishiki H, Ishiwada N, Fukasawa C, et al. Incidence of bacterial coinfection with respiratory syncytial virus bronchopulmonary infection in pediatric inpatients. J Infect Chemother . 2011;17:87-90.

51. Suarez-Arrabal MC, Mella C, Lopez SM, et al. Nasopharyngeal bacterial burden and antibiotics: Influence on inflammatory markers and disease severity in infants with respiratory syncytial virus bronchiolitis. J Infect . 2015;71:458-469.

52. Weinberger DM, Klugman KP, Steiner CA, Simonsen L, Viboud C. Association between respiratory syncytial virus activity and pneumococcal disease in infants: a time series analysis of US hospitalization data. PLoS Med . 2015;12:e1001776.

53. Kanmani P, Clua P, Vizoso-Pinto MG, et al. Respiratory commensal bacteria Corynebacterium pseudodiphtheriticum improves resistance of infant mice to respiratory syncytial virus and Streptococcus pneumoniae superinfection. Front Microbiol . 2017;8:1613.

54. Le Souef P. Viral infections in wheezing disorders. Eur Respir Rev . 2018;27:170133.

55. Leibovitz E, Raiz S, Piglansky L, et al. Resistance pattern of middle ear fluid isolates in acute otitis media recently treated with antibiotics. Pediatr Infect Dis J . 1998;17:463-469.

Figure legends 
Figure 1. Nasopharyngeal microbiome clusters in healthy infants and changes associated with age, acute respiratory infections (ARI), antibiotic treatment, increase morbidity and severe prematurity.

Figure 2. Nasopharyngeal microbiome modifications increasing the risk of RSV disease severity, delayed viral clearance and increased risk of recurrent wheezing.

Figure 3. Nasopharyngeal microbiome modifications induced or associated with mild or severe RSV infection promoting bacterial superinfections.
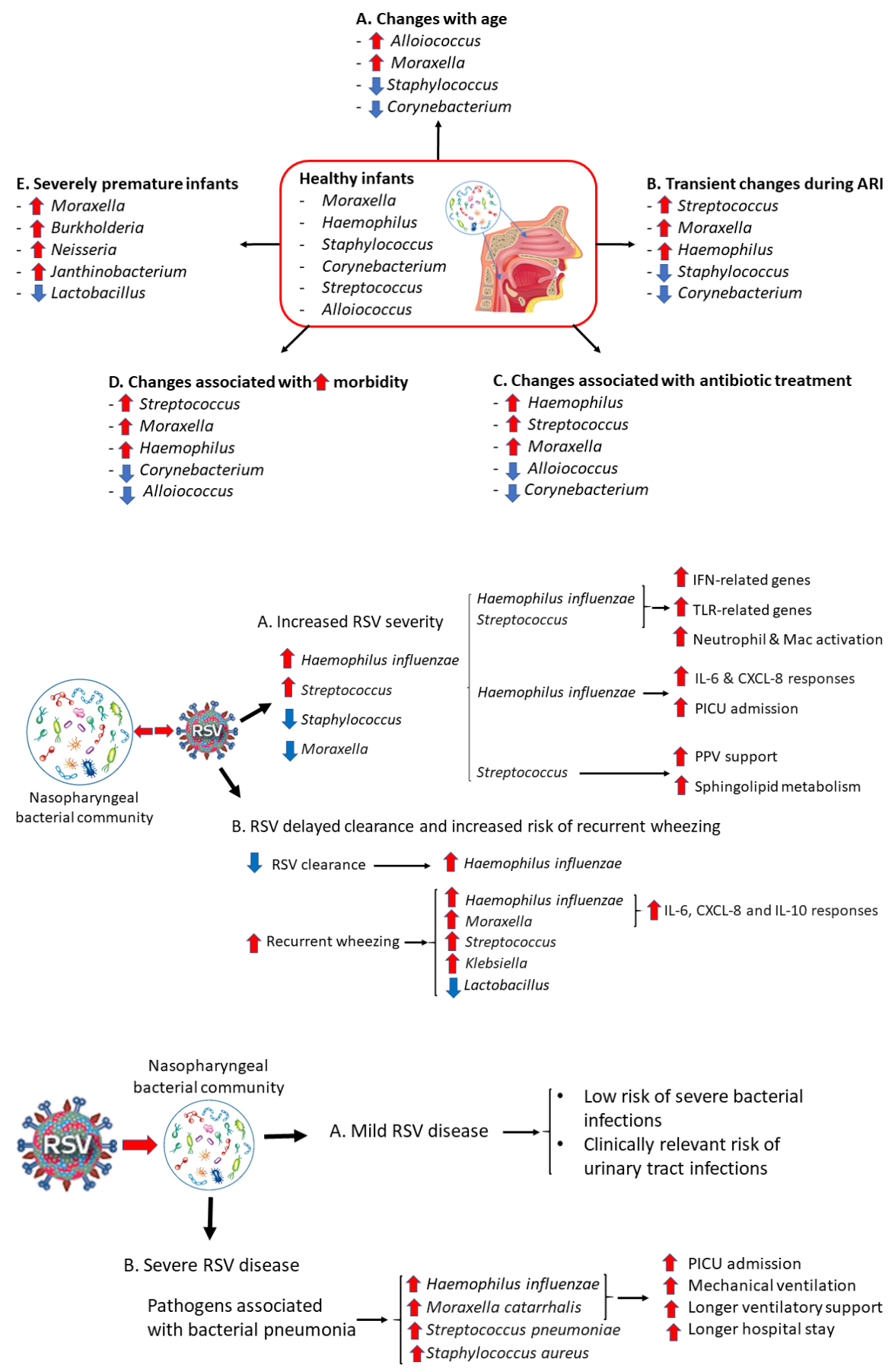